\title{
Symbolic-Numeric Computation of the Eigenvalues and Eigenfunctions of the Leaky Modes in a Regular Homogeneous Open Waveguide
}

\author{
Dmitriy Divakov ${ }^{1}$, Anastasiia Tiutiunnik ${ }^{1}$, and Anton Sevastianov ${ }^{1}$ \\ ${ }^{1}$ Peoples' Friendship University of Russia (RUDN University), 6 Miklukho-Maklaya St, Moscow, 117198, Russian Federation
}

\begin{abstract}
In this paper the algorithm of finding eigenvalues and eigenfunctions for the leaky modes in a three-layer planar dielectric waveguide is considered. The problem on the eigenmodes of open three-layer waveguides is formulated as the Sturm-Liouville problem with the corresponding boundary and asymptotic conditions. In the case of guided and radiation modes of open waveguides, the Sturm-Liouville problem is formulated for self-adjoint second-order operators on the axis and the corresponding eigenvalues are real quantities for dielectric media. The search for eigenvalues and eigenfunctions corresponding to the leaky modes involves a number of difficulties: the boundary conditions for the leaky modes are not self-adjoint, so that the eigenvalues can turn out to be complex quantities. The problem of finding eigenvalues and eigenfunctions will be associated with finding the complex roots of the nonlinear dispersion equation. In the present paper, an original scheme based on the method of finding the minimum of a function of several variables is used to find the eigenvalues. The paper describes the algorithm for searching for eigenvalues, the algorithm uses both symbolic transformations and numerical calculations. On the basis of the developed algorithm, the dispersion relation for the weakly flowing mode of a three-layer open waveguide was calculated in the Maple computer algebra system.
\end{abstract}

\section{Introduction}

The paper presents the formulation of the problem of numerical calculation of the leaky modes for planar three-layer waveguides.

The propagation of waveguide radiation in open waveguides is described by Maxwell's equations, boundary conditions at media interface, constitutive equations and asymptotic conditions [1-3].

The Maxwell's equations and the material equations in the Gaussian system of units have the following form [1]:

$$
\begin{aligned}
\operatorname{rot} \vec{E} & =-\frac{1}{c} \frac{\partial \vec{B}}{\partial t}, \\
\operatorname{rot} \vec{H} & =\frac{1}{c} \frac{\partial \vec{D}}{\partial t}, \\
\vec{D} & =\varepsilon \vec{E}, \\
\vec{B} & =\mu \vec{H},
\end{aligned}
$$

where $\vec{E}, \vec{H}$ - vectors of electric and magnetic field intensity; $\vec{D}, \vec{B}$ - vectors of electric and magnetic inductions; $c$ - speed of electromagnetic wave propagation in vacuum; $\varepsilon$ - dielectric permittivity, and $\mu$ - magnetic permeability of the medium.
At the interface of the dielectric waveguide layers, the boundary conditions imply the continuity of the tangential components of electromagnetic field $[2,3]$ :

$$
\left[\vec{E}^{\tau}\right]_{x=0, h}=\left[\vec{H}^{\tau}\right]_{x=0, h}=0,
$$

where $h$ - thickness of the waveguide layer, $[f]_{s=a}=f(a-0)-f(a+0)-$ jump of the function $f$ at the point $s=a$, and tangential components of the electromagnetic field $\vec{E}^{\tau}$ and $\vec{H}^{\tau}$ are defined as $\vec{E}^{\tau}=\vec{E} \times \vec{n}, \vec{H}^{\tau}=\vec{H} \times \vec{n}$ with the normal to the layers interface $\vec{n}$.

The asymptotic conditions for the leaky modes in planar waveguide structure have the following form:

$$
\begin{aligned}
& \frac{\partial E_{y}}{\partial x}-i k_{0} \sqrt{n_{c}^{2}-\beta^{2}} E_{y} \underset{x \rightarrow+\infty}{\longrightarrow} 0, \\
& \frac{\partial E_{y}}{\partial x}+i k_{0} \sqrt{n_{s}^{2}-\beta^{2}} E_{y} \underset{x \rightarrow-\infty}{\longrightarrow} 0,
\end{aligned}
$$

where $k_{0}$ - wavenumber in vacuum, $n_{c}$ - refractive index for the covering layer, $n_{s}$ - refractive index of the substrate.

This work considers an open, regular, planar waveguide with three layers: a covering layer, a waveguide layer (film) and a substrate. The thickness of the covering layer and the substrate is semi-infinite, 
while the thickness of the waveguide layer is comparable to the wavelength. The refractive index is a piecewise constant function:

$$
n(x)= \begin{cases}n_{c}, & x>h \\ n_{f}, & 0 \leq x \leq h \\ n_{s}, & x<0,\end{cases}
$$

where $n_{f}$ - refractive index of the waveguide layer.

The radiation propagates along $\mathrm{Oz}$ axis. The geometry of such a structure is shown in figure 1 .

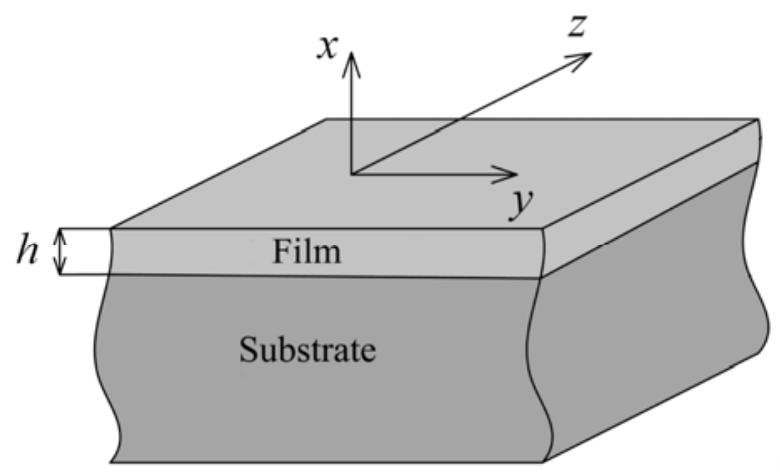

Fig. 1. Geometry of the dielectric waveguide structure

\section{The problem formulation}

For planar structures, time harmonic fields, under the assumption of the field invariance along the direction $O y$, the Maxwell's equations are reduced to a pair of the Helmholtz equations: TE-mode and TM-mode; and two pairs of additional relations [1-6]:

$$
\left\{\begin{array}{c}
\left(\left(\frac{\partial^{2}}{\partial z^{2}}+\frac{\partial^{2}}{\partial x^{2}}\right)+k_{0}^{2} \varepsilon(x) \mu\right) E_{y}=0 \\
H_{x}=-\frac{1}{i k_{0} \mu} \frac{\partial E_{y}}{\partial z} \\
H_{z}=\frac{1}{i k_{0} \mu} \frac{\partial E_{y}}{\partial x}
\end{array}\right.
$$

and

$$
\left\{\begin{array}{c}
\left(\frac{\partial^{2}}{\partial z^{2}}+\varepsilon(x) \frac{\partial}{\partial x}\left(\frac{1}{\varepsilon(x)} \frac{\partial}{\partial x}\right)+k_{0}^{2} \varepsilon(x) \mu\right) H_{y}=0 \\
E_{x}=\frac{1}{i k_{0} \varepsilon(x)} \frac{\partial H_{y}}{\partial z} \\
E_{z}=-\frac{1}{i k_{0} \varepsilon(x)} \frac{\partial H_{y}}{\partial x}
\end{array}\right.
$$

In this paper we consider only the TE-mode. Applying the separation of variables method, we obtain the equation for the transverse and longitudinal parts [49]

$$
\begin{gathered}
\psi^{\prime \prime}+k_{0}^{2} n^{2}(x) \psi=k_{0}^{2} \beta^{2} \psi, \\
U^{\prime \prime}=-k_{0}^{2} \beta^{2} U .
\end{gathered}
$$

In this paper we consider only the solution of the equation for the transverse part. We obtain the SturmLiouville problem:

$$
\left\{\begin{array}{c}
\psi^{\prime \prime}+k_{0}^{2} n^{2}(x) \psi=k_{0}^{2} \beta^{2} \psi \\
{\left.[\psi]\right|_{x=0, h}=\left.\left[\psi^{\prime}\right]\right|_{x=0, h}=0} \\
\psi^{\prime}+i k_{0} \sqrt{n_{s}^{2}-\beta^{2}} \psi \underset{x \rightarrow-\infty}{\longrightarrow} 0 \\
\psi^{\prime}-i k_{0} \sqrt{n_{c}^{2}-\beta^{2}} \psi \underset{x \rightarrow+\infty}{\longrightarrow} 0
\end{array}\right.
$$

Taking into account the structure of the three-layer waveguide, the solution of the equation for the transverse part can be written in an analytical form in each of the regions, and for regions $x>h$ and $x<0$ it is necessary to choose a solution that satisfies the asymptotic conditions. As a result, we can formulate a problem describing the eigenfunctions and eigenvalues corresponding to the leaky modes [10-12]:

$$
\left\{\begin{array}{c}
\psi^{\prime \prime}+k_{0}^{2} n_{f}^{2} \psi=k_{0}^{2} \beta^{2} \psi \\
\left(\psi^{\prime}+i k_{0} \sqrt{n_{s}^{2}-\beta^{2}} \psi\right)_{x=0}=0 \\
\left(\psi^{\prime}-i k_{0} \sqrt{n_{c}^{2}-\beta^{2}} \psi\right)_{x=h}=0
\end{array}\right.
$$

\section{Solution algorithm}

In this paper we will consider an algorithm for finding the eigenvalues and functions for the leaky modes in a dielectric waveguide.

The equation for the transverse part of the waveguide layer has an analytical solution:

$$
\psi(x)=A \mathrm{e}^{i k_{0} \sqrt{n_{f}^{2}-\beta^{2}} x}+B \mathrm{e}^{-i k_{0} \sqrt{n_{f}^{2}-\beta^{2}} x},
$$

where $\psi(x)$ corresponds to the solution for the waveguide layer. Substituting the solution form for the waveguide layer into the boundary conditions, we obtain a homogeneous system of linear algebraic equations (SLAE), represented as a product of the coefficient matrix $\mathbf{M}$ and vector of sought-for amplitude coefficients $\vec{A}$ :

$$
\mathbf{M}(\beta) \vec{A}=\overrightarrow{0},
$$

where $\vec{A}=\left(\begin{array}{ll}A & B\end{array}\right)^{T}$ and $\mathbf{M}(\beta)$ defined as

$$
\mathbf{M}(\beta)=i k_{0}\left(\begin{array}{cc}
p_{f}+p_{s} & p_{f}-p_{s} \\
p_{f} \mathrm{e}^{i k_{0} p_{f} h}-p_{c} \mathrm{e}^{i k_{0} p_{f} h} & p_{f} \mathrm{e}^{-i k_{0} p_{f} h}+p_{c} \mathrm{e}^{-i k_{0} p_{f} h}
\end{array}\right)
$$

and $p_{\alpha}=p_{\alpha}(\beta), \alpha=c, f, s$ are presented below

$$
p_{f}(\beta)=\sqrt{n_{f}^{2}-\beta^{2}}, p_{s}(\beta)=\sqrt{n_{s}^{2}-\beta^{2}}, p_{c}(\beta)=\sqrt{n_{c}^{2}-\beta^{2}}
$$


To determine the sought-for values $A$ and $B$ it is necessary to solve a homogeneous SLAE.

In order for the system to have a nontrivial solution, it is necessary the determinant of the coefficient matrix of the system to be zero:

$$
\operatorname{det} \mathbf{M}(\beta)=0 .
$$

Since the matrix of coefficients has complex numbers, the solutions of the equation can also be complex numbers. Therefore, we move to the complex plane. Accordingly, it is necessary to find the acceptable values of the phase deceleration factor $\beta$ : solutions of the equation and such complex numbers for which the imaginary and real parts of the determinant will be approximately equal to zero. To simplify the finding of $\beta$, we declare the function of squared module of the determinant, depending on two real numbers:

$$
f(x, y)=|\operatorname{det} \mathbf{M}(x+i y)|^{2} .
$$

Thus, the problem of finding $\beta$ reduces to finding the minima of a function that depends on two real variables. Use the optimization method: pattern search. Since we know that the eigenvalues of the guided modes lie in the region from $n_{s}$ to $n_{f}$, we assume that the real part of the eigenvalues of the leaky modes lies in the interval from 0 to $n_{s}$. So we construct on this interval a dense grid of initial approximations in order to apply the optimization method in each of them. The resulting problem can be formulated as follows

$$
f(x, y) \underset{x \in\left[0 ; n_{s}\right], y>0}{\longrightarrow} \min .
$$

Thus, having obtained all the values, we have to select only those that are not only minima of the function $f(x, y)$, but also are the zeros of this function. Naturally, they should not be repeated.

After finding all the eigenvalues, it is necessary to solve a homogeneous SLAE: find the sought-for amplitude coefficients. To do this, we use the modified Gauss method. This method allows us to find a nontrivial solution for matrices with the last zero row.

Each $\beta$ has its own vector of amplitude coefficients:

$$
\mathbf{M}\left(\beta_{j}\right) \vec{A}_{j}=\overrightarrow{0} \text {. }
$$

This algorithm was implemented in the computer algebra system Maple $[4,5,9,10]$. In the algorithm there is a function of the accuracy problem of calculations. The algorithm is available by the link: https://bitbucket.org/asdrevitskiy/bgcw-programs/.

\section{Numeric results}

Below we graphically represent the roots of the complex-valued function $\operatorname{det} \mathbf{M}(\beta)$ for a three-layer planar open waveguide characterized by $n_{c}=1, n_{f}=2.10, n_{s}=1.47, h=0.55 \mu m \quad$ and $\lambda=0.55 \mu \mathrm{m}$.

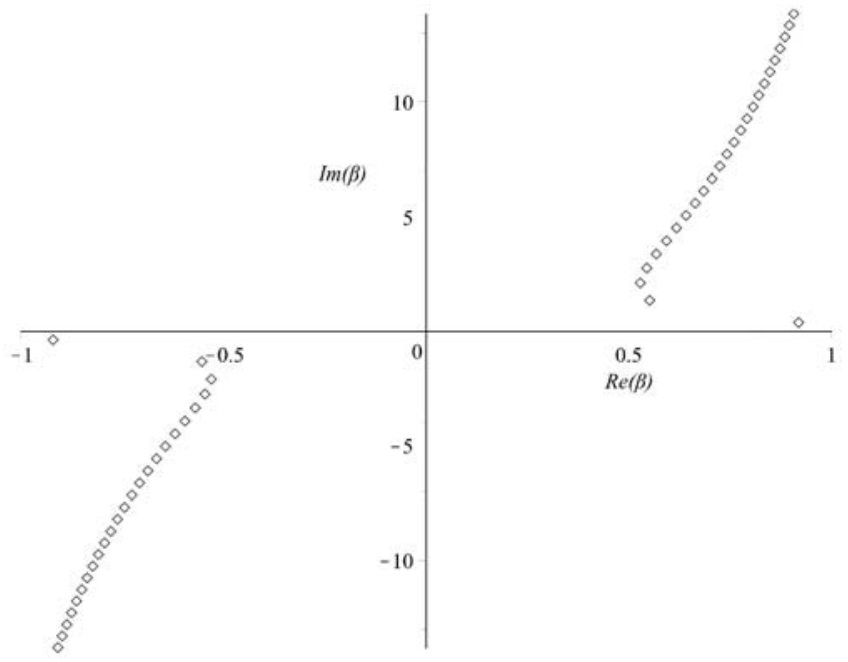

Fig. 2. Complex-valued coefficients $\beta$

As we can see from fig.2. there exists a complex eigenvalue with the smallest real part. The dispersion relation for leaky modes with the smallest real part of eigenvalues was calculated. For this purpose, the corresponding condition was written and integrated into the algorithm. The dispersion curves for real (Re) and imaginary $(\mathrm{Im})$ parts of these eigenvalues are shown in figures 3,4 .

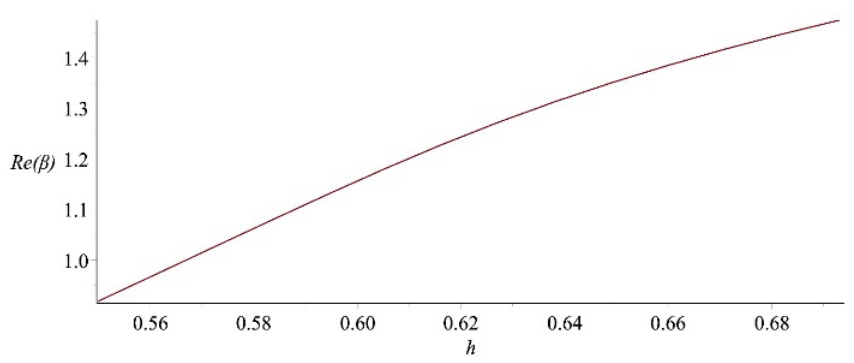

Fig. 3. Dispersion curve of the real (Re) part of $\beta$

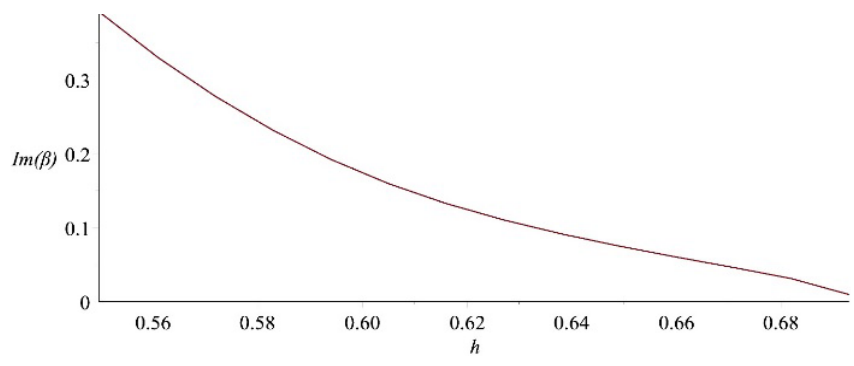

Fig. 4. Dispersion curve of the imaginary (Im) part of $\beta$

Also, for some of the eigenvalues (look for in Table 1) the eigenfunctions of the following modes were constructed, which are depicted in figures 5-8. 


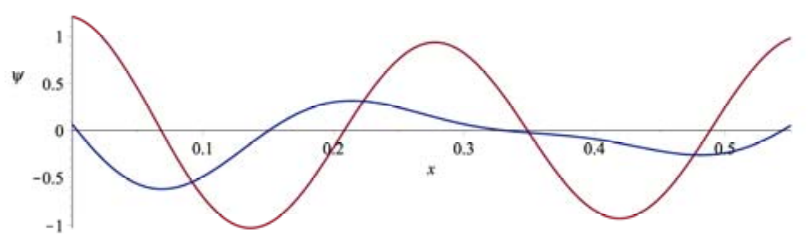

Fig. 5. Eigenfunction corresponding to eigenvalue $\beta=0.918713+0.391051 i$ (No.1 from Table 1)

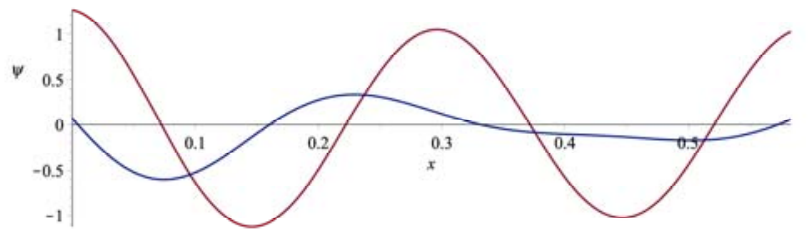

Fig. 6. Eigenfunction corresponding to eigenvalue $\beta=1.076580+0.231889 i$ (No.2 from Table 1)

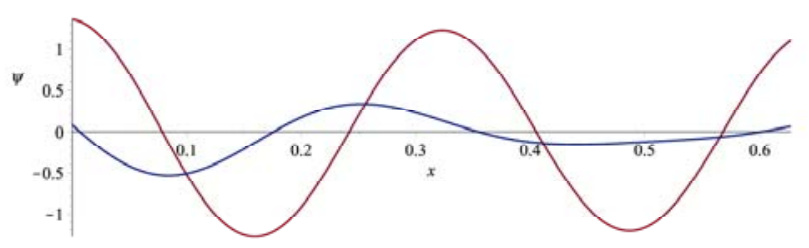

Fig. 7. Eigenfunction corresponding to eigenvalue $\beta=1.271199+0.110653 i$ (No.3 from Table 1)

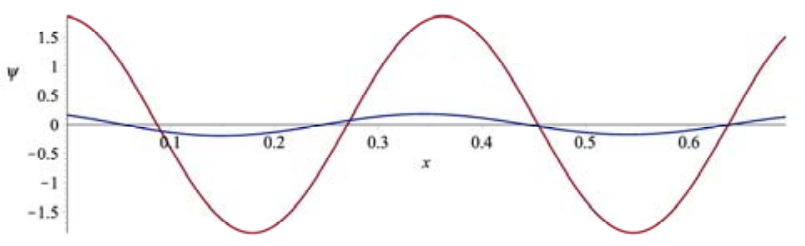

Fig. 8. Eigenfunction corresponding to eigenvalue $\beta=1.473693+0.009583 i$ (No.4 from Table 1)

Table 1. Eigenvalues

\begin{tabular}{|l|l|l|l|}
\hline$\#$ & $h$ & $\operatorname{Re}(\beta)$ & $\operatorname{Im}(\beta)$ \\
\hline 1 & 0.55 & 0.918713 & 0.391051 \\
\hline 2 & 0.583 & 1.076580 & 0.231889 \\
\hline 3 & 0.627 & 1.271199 & 0.110653 \\
\hline 4 & 0.693 & 1.473693 & 0.009583 \\
\hline
\end{tabular}

\section{Conclusion}

Within the framework of this paper, an algorithm for finding the eigenvalues and eigenfunctions of the leaky modes was presented. As a method of solution, the Hook-Jeeves method was chosen. Also, for the leaky modes, the dispersion dependence of the eigenvalues with the minimal imaginary part of the thickness of the waveguide layer was calculated.

The publication has been prepared with the support of the "RUDN University Program 5-100".

\section{References}

1. D. Marcuse, Light Transmission Optics, (Van Nostrand Reinhold, 1972).

2. M.J. Adams, An Introduction to Optical Waveguides, (John Wiley \& Sons, New York, Wiley, 1981).

3. T. Tamir, Guided-Wave Optoelectronics, (SpringerVerlag Berlin Heidelberg, 1988).

4. D. Divakov, M. Malykh, L. Sevastianov, A. Sevastianov, E. Ayryan, EPJ Web of Conferences, 173, 02007, (2018).

5. A.S. Drevitskiy, L.A. Sevastianov, D.V. Divakov, CEUR Workshop Proceedings, 1995, 22-27, (2017).

6. A.A. Egorov, L.A. Sevast'yanov, A.L. Sevast'yanov, Quantum Electronics, 44, 2, 167-173, (2014).

7. M.D. Malykh, L.A. Sevastianov, A.A. Tiutiunnik, N.E. Nikolaev, Journal of Electromagnetic Waves and Applications, 32, 7, 886-898, (2018).

8. M. Malykh, L. Sevastianov, A. Tyutyunnik, N. Nikolaev, EPJ Web of Conferences, 173, 02014, (2018).

9. L.A. Sevastianov, A.A. Egorov, A.L. Sevastyanov, Physics of Atomic Nuclei, 76, 2, 224-239, (2013).

10. F. Emami, F. Hoseini, International Journal of Fundamental Physical Sciences, 3, 2, 11-15, (2013).

11. S.K. Raghuwanshi, V. Palodiya, Sensor Letters, 13, 9, 735-742, (2015).

12. A.B. Sotsky, L.M. Steingart, J.H. Jackson, P.Ya. Chudakovskii, L.I. Sotskaya, Technical Physics, 58, 11, 1651-1660, (2013). 